\title{
Portable Freehand System for Real-time Antenna Diagnosis and Characterization
}

\author{
Guillermo Álvarez-Narciandi, Jaime Laviada, Yuri Álvarez-López, Senior Member, IEEE, and Fernando \\ Las-Heras, Senior Member, IEEE
}

\begin{abstract}
This paper presents an agile system for antenna diagnosis. The system is operated by means of freehand movements of the probe antenna avoiding the need of bulky positioners and thus providing portability to the system. For that purpose, the probe antenna position, which is required by the antenna diagnosis algorithm, is retrieved by means of an affordable and quickly-deployable motion capture system. The probe position and the acquired field are used to characterize the antenna under test by computing an equivalent currents distribution on the antenna aperture and its far-field pattern in real-time. To achieve that, an ad hoc workflow is designed including a method for spatially balancing the acquired field samples. Although the system is not intended to provide an accuracy comparable to the one achieved by anechoic chamber facilities, it opens a new horizon of possibilities for in situ agile characterization of antennas enabling the detection of faulty elements or radiation pattern deviations. Results at $\mathrm{Ku}$ and $\mathrm{Ka}$ bands, supported by attached multimedia material, are presented to illustrate the capabilities of the system.
\end{abstract}

Index Terms-Antenna measurements; antenna diagnosis; freehand; equivalent currents; sources reconstruction method; mm-wave antenna.

\section{INTRODUCTION}

A NTENNA diagnosis aims to detect malfunctions of radiating systems so the corresponding repair actions can be launched. This approach has been widely studied in last decades resulting in a large number of robust methods with different scopes.

On one hand, a large family of antenna diagnosis techniques is devoted to the detection of faulty elements by analyzing the impact of one or more failures in the far-field pattern [1], [2]. Since the number of faulty elements is usually expected to be low, this kind of problem can be formulated as a compressed sensing problem taking advantage of the benefits of this technique with respect to sampling [3]. In addition, similar techniques exploiting near-field rather than far-field observations are also available [4]. Although the number of techniques in this family is large, the general advantage is that they can operate with a small number of samples as well as their computational efficiency. The drawbacks are: i) the limited amount of information provided by these techniques as they only provide information about status of elements,

This work has been partially supported by the Ministerio de Ciencia, Innovación y Universidades of Spain /FEDER under project RTI2018-095825B-I00, by the Gobierno del Principado de Asturias under project GRUPINIDI-2018-000191 and under the FPU grant FPU15/06431.

The authors are with the Department of Electrical Engineering, University of Oviedo, Gijón, 33203, Spain. (e-mail: alvareznguillermo@uniovi.es; laviadajaime@uniovi.es; alvarezyuri@uniovi.es; flasheras@uniovi.es). which is usually reduced to a binary observation (i.e., healthy or not); ii) a potential relatively wide angular scan can be required when operating in the far-field, which is not always possible.

On the other hand, another large family of techniques exploits the Huygens' principle to reconstruct an equivalent currents distribution at a surface enclosing the antenna under test (AUT). These equivalent currents radiate the same fields as the AUT at any point outside the surface enclosing it [5], [6], [7]. In some cases (e.g. planar antennas, aperture antennas) the surface enclosing the AUT can be truncated to a finite size plane, as most of the energy radiated by the AUT is contained in such plane [8].

In the last years, several equivalent currents-based techniques have been implemented, due to their potential for antenna diagnosis and characterization using arbitrary-geometry measurement and reconstruction domains [6], [9]. Apart from antenna diagnosis, these techniques have been proved to be efficient for removing unwanted structural interactions [10], [11].

In this contribution, the Sources Reconstruction Method (SRM) described in [8] and [5] will be considered. This inverse problem is solved by expanding the unknown currents into known basis functions, whose coefficients are related to the field observations by means of a linear system of equations.

In contrast to the faulty element detection methods, the equivalent currents-based techniques provide information about the amplitude and phase of the fields around the antenna. Moreover, the far-field radiation pattern can be calculated from the reconstructed equivalent currents by means of a near-field to far-field transformation. Consequently, these techniques are able to detect the amplitude and phase of each element of the antenna as well as other undesired effects such as unwanted reflections in the antenna itself or its neighborhood. This insightful information comes at the expense of computationally intensive algorithms to solve the system of equations. However, this last point has been alleviated by means of several approaches such as the fast multiple method (FMM) [12], [13], the adaptive cross algorithm (ACA) [14] or the use of GPUs [15].

Despite the robustness of the previous diagnosis approaches, these techniques usually resort to relatively bulky and heavy equipment in order to provide reliable sampling of the radiated fields. This paper aims to present an agile diagnosis system, exploiting the basis of some of the previous approaches, to provide real-time diagnosis from freehand movements so that the system can bypass the need of heavy positioners. The 
system is mostly intended to work at mm-wave frequencies at which the impact of a non-anechoic environment is in general significantly smaller than at lower frequencies. Although it clearly has an impact in the accuracy, it will be shown that the system is still able to accurately detect the status of the elements and provide a fair estimate of the far-field radiation pattern.

Regarding the kind of diagnosis method, the source reconstruction, conveniently modified to consider the particularities of the system, is used as it provides the more complete information about the amplitude and phase distribution of the antenna as well as its radiation pattern.

The proposed system entails a number of challenges. The first one is the physical implementation as it requires an accurate position tracking as well as portable components. However, current technologies in terms of affordable infrared cameras, providing position reports with an accuracy in the order of $\sim 100-200 \mu \mathrm{m}$, as well as portable VNAs make possible to easily implement the system by means of commercial components. In particular, the idea of increasing the flexibility of the acquisition schemes by means of using camera-based positioning systems has been presented in [16] and [17] to directly map either the electric or the magnetic field strength radiated by a device under test onto its predefined 3D model manually. In addition, the same authors also proposed the use of a camera-based positioning system to track a probe under 2D constrained movements in order to map electromagnetic sources by means of backpropagation in [16] and [18]. Although this system improves the flexibility with respect to conventional mechanical acquisition systems, it imposes several constraints that limit its applicability in some scenarios. In particular, it requires a planar structure made of electrically transparent material (such as foam) to ensure a planar scan and a gimbal stabilizer to maintain the orientation of the scanning probe. In addition, it uses an FFT-based method, which requires a regular acquisition grid (missing points are set to zero). The use of a motion capture system in the context of radar imaging was presented in [19].

The second major challenge comes from the fact of acquiring data irregularly sampled due to the freehand scanning. Most of source reconstruction methods are intended to work with data coming from regular grid acquisitions such as planar [20], cylindrical or spherical [6], [21] acquisitions. However, it is relevant to observe that irregularly sampled data has been recently considered by some diagnosis methods to take into account possible deviations from a regular grid, i.e., positioner errors that can be tracked. In particular, quasi-cylindrical, spherical and -planar data has been studied for diagnosis and near-field to far-field transformations based on plane or spherical wave expansions [22], [23], [24], which can also be formulated in terms of a linear system of equations but relating electromagnetic modes rather than equivalent currents (see [25] for a detailed comparison). In the specific framework of source reconstruction, irregular sampling has been considered on systems based on a large gondola-positioner in which accurate position control is not possible [26] and on systems based on unmanned air vehicles [27], [28]. However, none of the previous works are focused on dealing with the kind

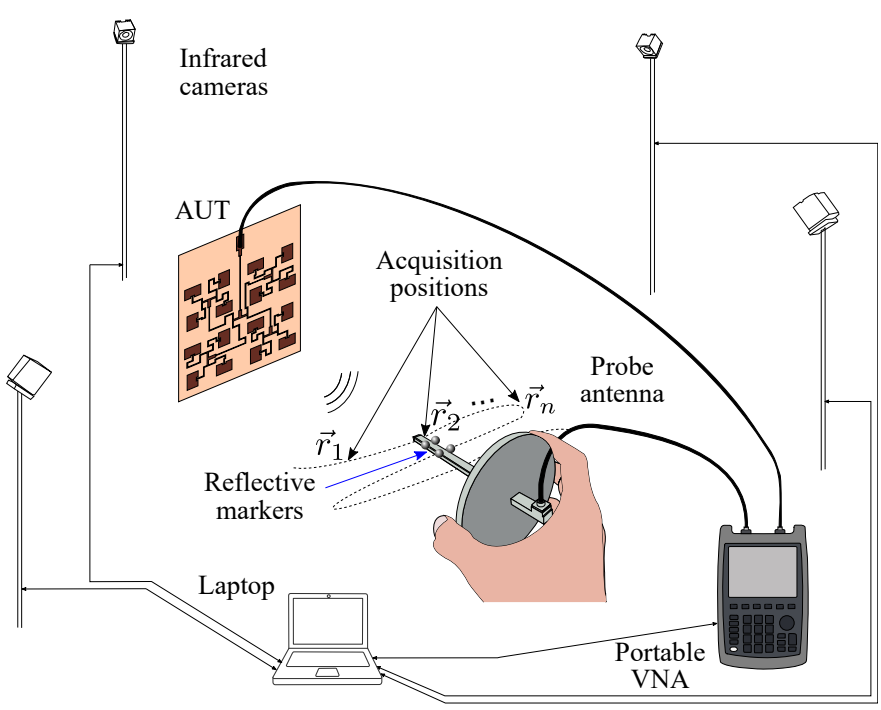

Fig. 1. Scheme of the proposed system.

of irregularities which can appear when performing freehand scanning.

The main contribution of this manuscript, which extends the conference paper presented in [29], is a setup to perform freehand antenna diagnosis complemented by a modified source reconstruction method to provide real-time results which takes into account the irregular sampling due to the freehand scanning.

This paper is organized as follows. First, the system architecture is presented and the workflow of the system is described in Section II. Validation of the system with measurements from different AUTs is presented in Section III, discussing and comparing them with those obtained using a planar range. Finally, the conclusions are drawn in Section IV.

\section{DESCRIPTION OF THE SYSTEM}

The presented system aims to characterize an antenna under test by means of the equivalent sources computed on a surface enclosing the AUT. The sources are computed from near-field samples measured at arbitrary positions by means of freehand movements of the probe antenna. The relevant hardware components, the system flowchart and the modified source reconstruction method are described next.

\section{A. Hardware}

The main components of the system are depicted in Fig. 1. The system comprises three major subsystems: i) electromagnetic field acquisition subsystem; ii) positioning subsystem; iii) processing subsystem. In general, commercial off-the-shelf components will be used for the implementation.

The electromagnetic field acquisition subsystem comprises a probe antenna and a vector network analyzer (VNA). As in conventional antenna measurements, the VNA is connected to the AUT as well as to the probe antenna (e.g., an open-ended waveguide) to acquire the $S_{21}$ parameter. In the presented implementation, two different VNAs have been used. The first 
one is a Fieldfox N9950A, which is a portable VNA with capabilities to perform measurements up to $14 \mathrm{GHz}$. The second VNA is a Keysight PNA-X, which provides better capabilities in terms of dynamic range, time sweep and frequency range at the expenses of larger dimensions and weight in line with standard laboratory analyzers.

In order to perform the antenna diagnosis, it is necessary to know the radiated field but also the position at which the field samples are acquired. Thus, the second major component of the system is the positioning subsystem. This subsystem is devoted to acquire both the position and attitude of the probe antenna. The positioning subsystem must meet several requirements. First, it must be compatible with the probes so it does not require significant, if any, modifications of the probe. Second, it must be accurate enough so that positioning error has a low or negligible impact in the final result. The latter criteria is met as long as the positioning error is much smaller than the working wavelength. Third, the positioning system must provide real-time position information.

In the implementation presented in this contribution, a motion capture system [30] has been adapted to work as positioning subsystem. The system comprises four infrared cameras at fixed positions and four markers with a diameter of $6.5 \mathrm{~mm}$, which can be easily attached to most of probes. The system can be easily calibrated in a few minutes by means of its own calibration software and moving a wand with markers at known positions. The system provides up to 240 positions per second, which is enough for a real-time system. In our setup, the calibration shows an error in the range of $100-200 \mu \mathrm{m}$. Thus, this error is expected to have a negligible impact in the final results up to $15 \mathrm{GHz}$ if the criteria of $\lambda / 100$ is assumed as in IEEE standards for antenna measurements [31], [32]. However, since other sources of error are expected to have a larger impact (e.g., reflections due to the lack of an anechoic environment), the positioning error can be relaxed. In particular, under a criterion of $\lambda / 10$, the setup is expected to provide fair results up to $150 \mathrm{GHz}$.

The third pillar of the presented system is the processing subsystem. This subsystem processes the acquired radiated field and positions by means of a source reconstruction method (SRM) to obtain the antenna diagnosis information. In addition, it also triggers the synchronous acquisition of the position and radiated field. The flowchart of this processing is detailed later in section II-B. This processing subsystem is implemented by means of one or two conventional PCs ${ }^{1}$ since the sample acquisition and the SRM can be run in independent computers to alleviate the computational cost.

\section{B. Flowchart}

The system is based on continuously moving the probe antenna over the antenna under test so that the radiated field is sampled at different positions. During this process, the system continuously updates the antenna characterization in terms of equivalent currents in the aperture and of far-field pattern. Those functionalities are implemented by means of the flowchart shown in Fig. 2.

\footnotetext{
${ }^{1}$ In general, laptops are preferred due to portability concerns.
}

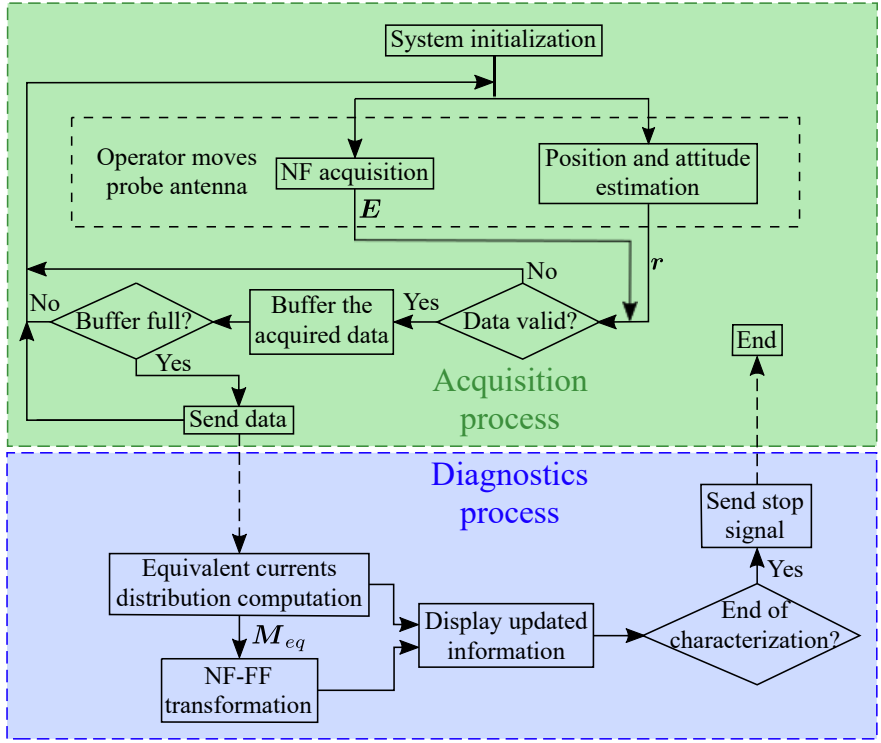

Fig. 2. Workflow of the proposed system.

The workflow involves two independent processes, which can be run in different machines, both in parallel to the operator movements. The acquisition process is in charge of the synchronous acquisition of the position and field samples. In order to balance the sample distribution, the approach detailed in section II-D is used, resulting in some discarded samples based on the acquisition position. In addition, in order to ensure that the main lobe of the probe antenna points to the front as in a conventional planar range and that the desired polarization is measured, the orientation of the probe antenna is monitored and measurements when the yaw, pitch or roll angles of the probe are above a given threshold are also discarded. According to the performed measurements, a threshold value of $5^{\circ}$ provides a good trade-off between scanning speed and accuracy. Furthermore, in order to set an upper bound of the probe movement during a frequency sweep, the position of the probe is queried before and after the sweep and, if the difference is higher than a given frequencydependent threshold, the sample is discarded. In particular, for the measurements discussed in this paper this threshold was set to $1 \mathrm{~mm}$ in the case of the mm-wave frequency measurements and to $2 \mathrm{~mm}$ in the case of the X-band measurements. Nondiscarded samples are buffered until a certain amount of samples is gathered and, after that, the samples are sent to the diagnosis process. The sample buffer avoids an unnecessary computational burden associated to recomputing the equivalent currents for each new acquisition as the operator usually does not require an update rate so high.

The diagnosis process receives and stores the position and field samples. After receiving each set of samples, it computes the equivalent currents distribution and the radiation pattern of the AUT (see next section for further details) while more data is acquired by the operator. The diagnosis information (currents distribution and radiation pattern) is updated and displayed in real-time, alongside with a heatmap depicting where measurements have been acquired. In addition, the 


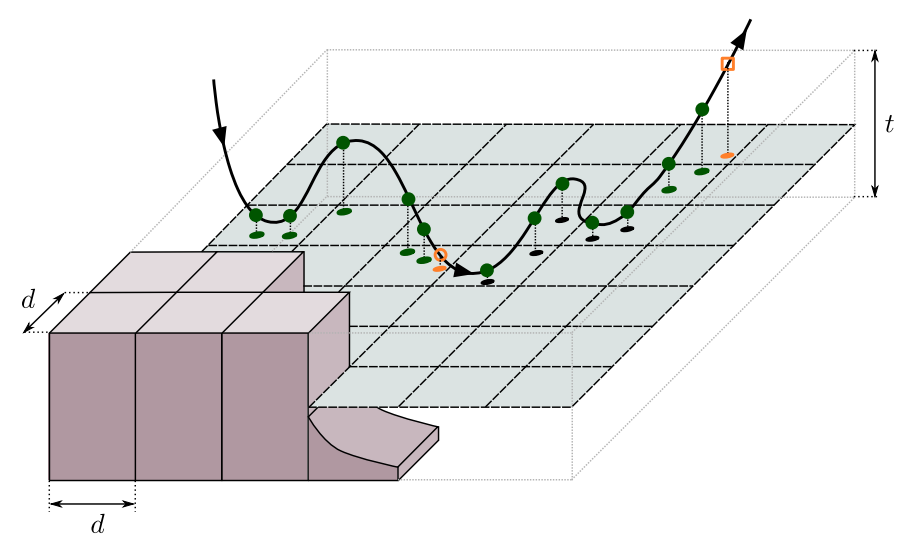

Fig. 3. Sampling distribution balance by setting maximum number of samples per cell. In order to help in the visualization of the trajectory and sampling position, only a few cells are shown together with the center of the cells. Orange samples are discarded either because the maximum number of samples is exceeded (e.g., the empty orange circle sample) or because the maximum vertical tolerance is exceeded (e.g, the empty orange square sample). A maximum of two samples per cell is considered.

amplitude and phase of the acquired fields are shown on a bidimensional grid. All the previous real-time information is intended to aid the operator to decide how to proceed to scan. For example, the operator can dynamically change from scanning a certain area to a different one based on the achieved sample density, the lack of impact of new samples in the final result, energy of the sampling area, etc.

\section{Source Reconstruction Method}

The acquired field and position samples are processed by means of a source reconstruction method (SRM) [5]. In the presented implementation, a planar reconstruction domain (usually placed at the antenna aperture) is chosen and, therefore, only the equivalent magnetic currents are needed [8]. The magnetic currents are discretized by means of known basis functions yielding the following linear system of equations:

$$
\overline{\bar{A}}\left[\begin{array}{c}
b_{1} \\
b_{2} \\
\vdots \\
b_{N}
\end{array}\right]=\left[\begin{array}{c}
E_{1} \\
E_{2} \\
\vdots \\
E_{M}
\end{array}\right]
$$

where $b_{n}$ is the weight of the $n$-th basis function and $E_{m}$ is the field sample at the $m$-th considered position. The matrix $\overline{\bar{A}}$ has dimensions $M \times N$ where $N$ is the number of basis functions and $M$ is the number of considered NF samples. The elements at row $m$ and column $n$ of matrix $\overline{\bar{A}}$ relate the field at the $m$-th position radiated by the $n$-th basis function with the corresponding evaluation of the Green's function [5].

The previous system of equations, which is usually overdetermined, is solved by means of an iterative solver, such as the conjugated gradient method, providing the minimum error and energy solution to (1). After that, the far-field radiation pattern is computed by using the corresponding radiation integrals [33].

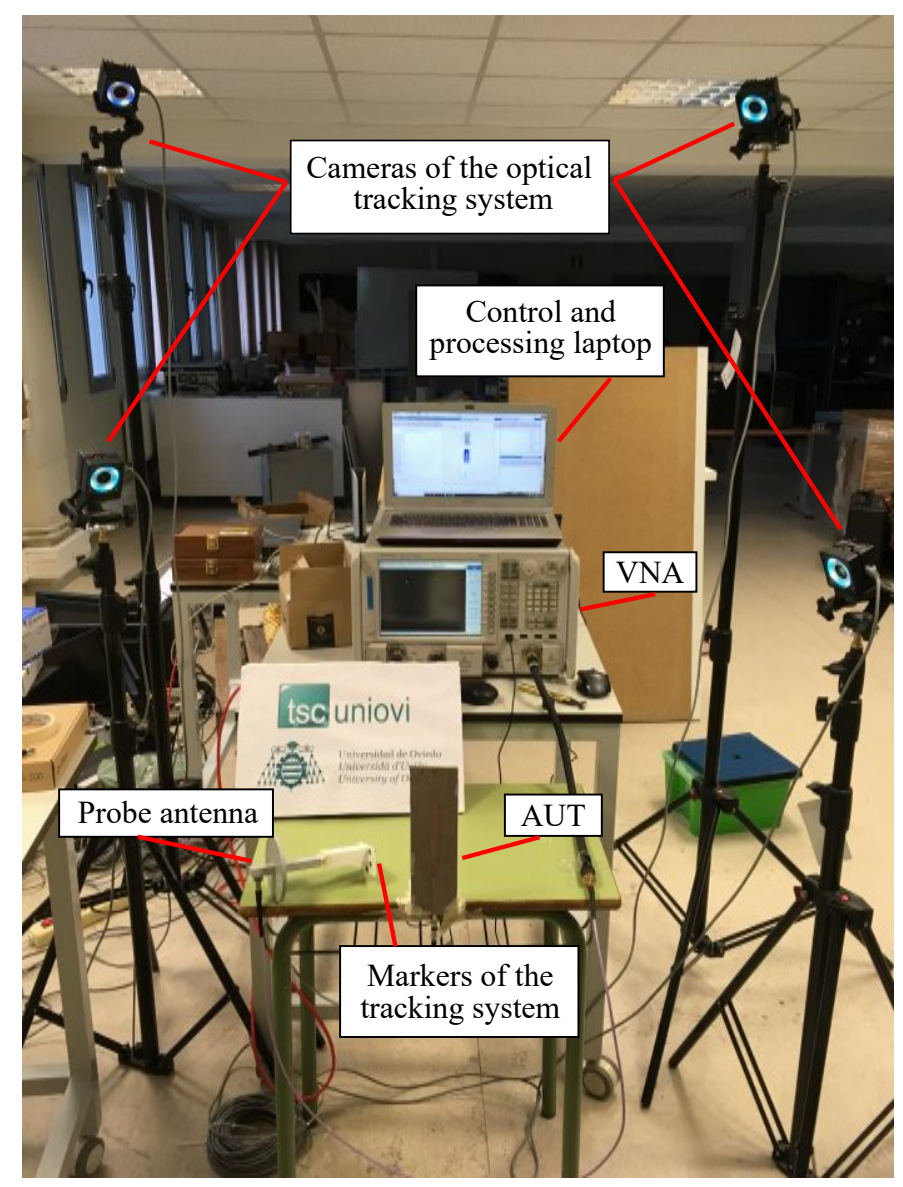

Fig. 4. Measurement setup.

\section{Sampling distribution balance}

The acquisition process is continuously registering positions and field samples. This fact, together with the non regular hand movements, will result in an uneven sampling distribution. For example, if the operator stops (or moves slowly) at a certain area, the system would keep sampling yielding a high number of samples for a small region. This kind of locally oversampled areas can result in an anomalous solution of (1) due to the higher weight that would be given to them. For this reason, an approach to locally balance the sample distribution is required.

In this work, the uneven sampling problem is solved by limiting the number of local samples per given surface area. This is implemented by splitting the observation volume, i.e., the volume in which the samples are acquired, into nonoverlapped cells and setting a maximum number of samples per cell $P$. In particular, only the first $P$ samples acquired inside a given cell are considered so any additional acquisition in that cell is discarded. The system of equations given in (1) can be easily implemented by updating the matrix $\overline{\bar{A}}$ with new rows corresponding to these new acquisitions.

In our implementation, the cells are chosen so that the acquisition macroscopically mimic a conventional planar range acquisition. In particular, cells are shaped as rectangular cuboids with dimensions $d \times d \times t$ (see Fig. 3). On one hand, the horizontal dimensions $d$ of the cuboids are intended to play the role of the conventional sampling steps and, therefore, 


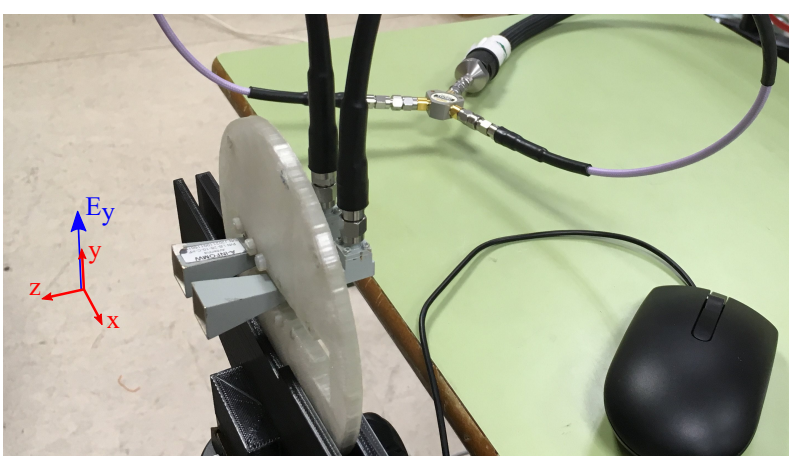

Fig. 5. Measured two horn antenna array.

they should be in the order of $\lambda / 2$. However, this size can be relaxed if more than one sample per cuboid is considered. On the other hand, the vertical cell size $t$ enables some tolerance so that it relaxes the operator movement and it is usually chosen up to $\lambda / 2$ to avoid electrically large gaps between adjacent samples. It should be pointed out that when more than one acquisition per cell is performed, i.e. $P>1$, the effective sampling step in the vertical dimension would be smaller than the value of the tolerance, $t$, and, thus, the requirement of $t<\lambda / 2$ can be relaxed. Since samples outside those cells are discarded, a visual aid is included in the interface so that the operator knows the distance to the cells bottom and top in order to move the probe to the corresponding area. In addition, the density of samples per cell is shown by means of the aforementioned heatmap.

An example of the use of cells is shown in Fig. 3. In this example, only a few cuboids are shown to ease the visualization of the trajectory of the probe and the acquired samples, but a plane through the center of the cells is shown to help in illustrating the process. In this example, a maximum of two samples per cell (i.e., $P=2$ ) is considered. Thus, the sample corresponding to the orange empty circle marker is discarded since two samples are already available. In addition, the sample corresponding to the orange empty square marker is also discarded because the vertical tolerance is exceeded.

In addition to mitigate the effect of non-equally spaced samples, it is important to note that there are some environmental agents that can distort the measurement. In particular, these agents are: a) coupling between the AUT and the probe as well as between the probe and the operator holding the antenna; $b$ ) reflections due to the non-anechoic environment.

The reactive coupling between the AUT and the probe follows the same rules as in conventional planar measurements and therefore it is considered that the near-field acquisition can be successfully performed as long as a distance of a few wavelengths is considered [34]. It is relevant to consider that, as in conventional planar range measurements, the distance between the AUT and the acquisition plane (i.e., the reference surface) also has an impact on the plane truncation error [34].

Regarding the coupling between the probe and the person who is holding it (i.e., touching the probe antenna), a coupling similar to the supporting structures such as the ones in conventional facilities (e.g., an anechoic chamber) is expected. In this regard, the use of electrically long open-ended waveguides
(OEWG) is recommended in order to reduce reactive coupling.

Finally, the reflections due to the non-anechoic environment (including the operator) are also expected to have an impact in the accuracy of the system. These reflections are inherent to any in situ measurements. However, the impact of these reflections is expected to be significantly alleviated at high frequencies due to the propagation losses. As it will be experimentally shown, a good quality is found at mm-wave frequencies, whereas the system still shows a fair quality at a few $\mathrm{GHz}(12 \mathrm{GHz})$.

\section{VALIDATION OF THE SYSTEM}

The performance of the proposed system was assessed through several measurements conducted at the research facilities of the Signal Theory and Communications group of the University of Oviedo (TSC-UniOvi). A general overview of the setup is depicted in Fig. 4. The first set of tests consisted of different measurements of a two horn antenna array at Ka band and using the laboratory VNA (Keysight PNA-X) to measure the radiated signal. During the second set of tests a leaky wave antenna at $12 \mathrm{GHz}$ was characterized using both the aforementioned VNA and a portable VNA (Keysight Fieldfox N9950A). The sweep time of the PNA-X, which was configured to measure 201 frequency points in all of the measurements, was $1.45 \mathrm{~ms}$ and that of the Fieldfox, which was configured to measure 11 frequency points, was $24 \mathrm{~ms}$. The reference results of each set of measurements are compared with the ones obtained with a planar range of the University of Oviedo.

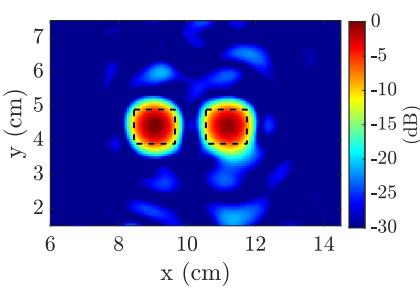

(a)

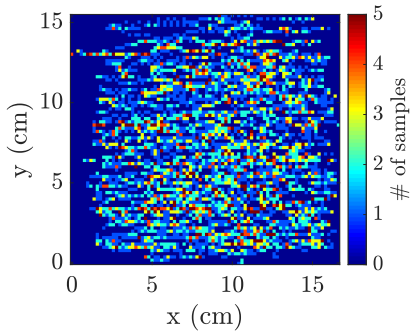

(c)

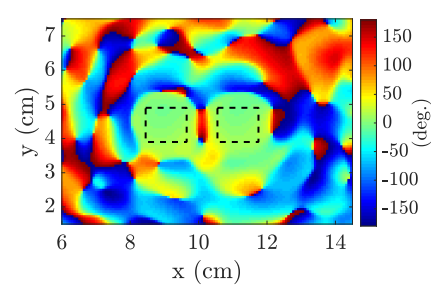

(b)

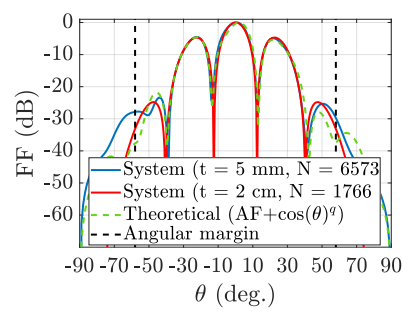

(d)
Fig. 6. Magnitude (a) and phase (b) of the equivalent currents distribution retrieved from the NF measurements, which were acquired at the positions depicted in the heatmap (c). The H-plane cut of the far-field pattern computed through a NF-FF transformation is shown in (d).

\section{A. Two horn antenna array}

First, a two horn antenna array at Ka band with the antennas separated $2.1 \mathrm{~cm}$ (Fig. 5) was measured. Four markers were attached by means of a $3 \mathrm{D}$ printed support structure to the 
probe antenna, which was an open-ended waveguide WR28, so that it could be tracked by the motion capture system.

A total of 6573 measurements were taken at an average distance of $3.5 \mathrm{~cm}$ from the AUT and the size of the cuboids used to balance the sampling was given by $d=2 \mathrm{~mm}$ and $t=5 \mathrm{~mm}$. The total acquisition time was $892 \mathrm{~s}$. The magnitude and the phase of the computed equivalent currents distribution at $32 \mathrm{GHz}$ are shown in Figs. $6 \mathrm{a}$ and $6 \mathrm{~b}$, respectively. As it can be seen, two similar sources both in phase and magnitude, corresponding to the two horn antennas of the array, can be clearly identified (the area corresponding to the aperture of each horn is enclosed by a black dashed line). The maximum of both sources differ only in $0.58 \mathrm{~dB}$ in magnitude and $1.2^{\circ}$ in phase (which may result from small imperfections in the setup of the array), and the separation between sources matches the physical space between both horns. The phase variation across the aperture of the horns is $27.9^{\circ}$. The heatmap, depicting where measurements were taken, is shown in Fig. 6c. Finally, the H-plane of the far-field pattern obtained from the equivalent currents distribution after performing a NF-FF transformation is shown in blue in Fig. 6d. The theoretical pattern, depicted in red, was obtained considering an array of two elements separated the same distance as the actual hornarray, fed with equal magnitude and phase and considering an element radiation pattern of $\cos ^{5}(\theta)$. The side lobes of the H-plane cut of both patterns are at $\pm 22^{\circ}$, being the correspondence between the theoretical model and the pattern obtained from the measurements very good within the angular margin of validity of the measurements.

In addition, the same AUT was measured using the proposed system but increasing the height of the cuboids (i.e. its dimension in the measurement direction) used to control the sampling to $t=2 \mathrm{~cm}$. In this case measurements were acquired at an average distance of $6.1 \mathrm{~cm}$ from the AUT. The computed H-plane of the far-field pattern is depicted in dashed-green line in Fig. 6d. As it can be seen, although the obtained pattern is slightly different, the accuracy is still sufficient for a fast diagnostic and characterization of an AUT outside a laboratory facility such as an anechoic chamber. In addition, relaxing the control of the sampling allows for a much faster diagnostic of antennas (the total scanning time for $t=2 \mathrm{~cm}$ was reduced to only $223 \mathrm{~s}$ ).

The same array was deployed inside a conventional planar range at the University of Oviedo [35] and it was measured using both the planar range and the proposed system to compare the obtained results with the reference ones retrieved using conventional techniques. Nevertheless, some cables were replaced resulting in slightly different phase shifts and, consequently, radiation patterns. The measurement setup is depicted in Fig. 7, where it is possible to observe the two-horn antenna array inside the planar range and the cameras of the motion capture system. During the freehand scan, a total of 4091 samples were acquired in an area of $19.6 \times 23.3 \mathrm{~cm}$ at $7.1 \mathrm{~cm}$ from the AUT in $984 \mathrm{~s}$. Using the planar range, a total of 3135 acquisitions were performed in a regular grid of $24.3 \times 25.2 \mathrm{~cm}$ at $10 \mathrm{~cm}$ from the AUT. The step between consecutive samples was $\lambda / 2$ at $32 \mathrm{GHz}$ and the total acquisition time was $6173 \mathrm{~s}$.

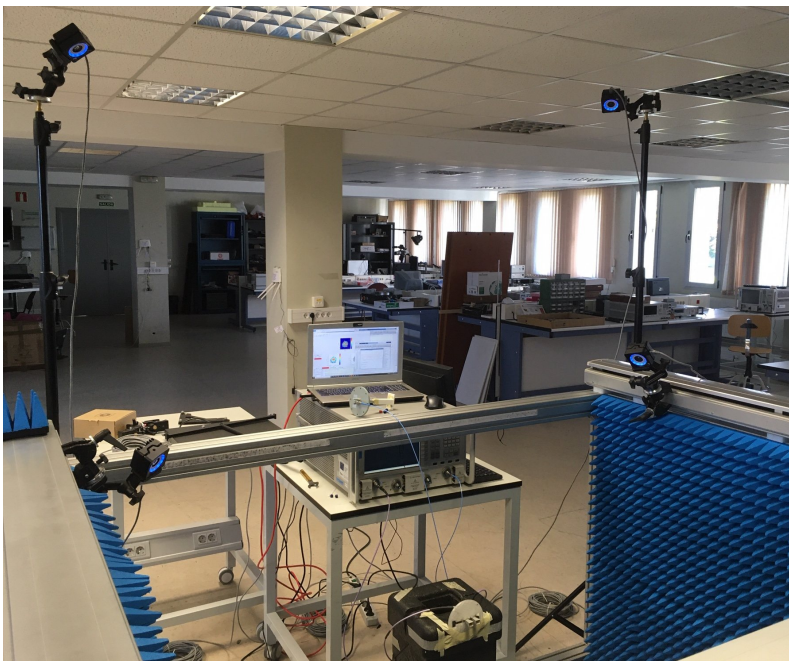

Fig. 7. Measurement setup in the planar range.

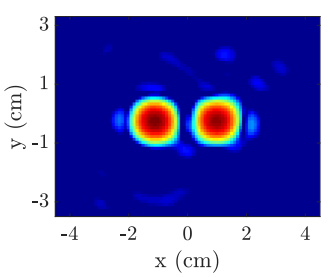

(a)

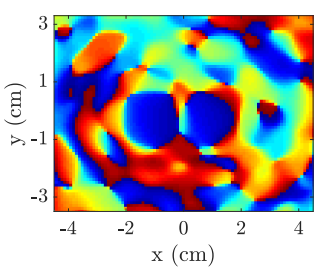

(c)

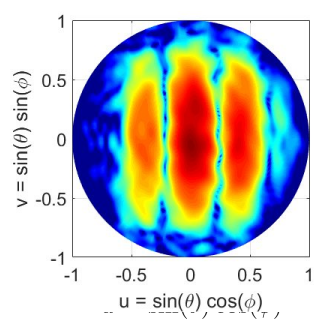

(e)

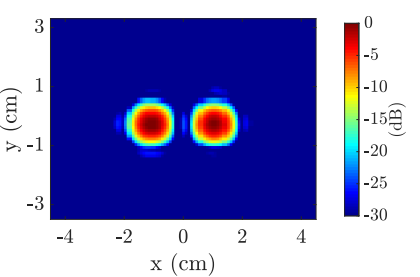

(b)

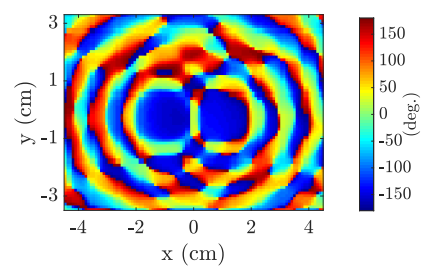

(d)

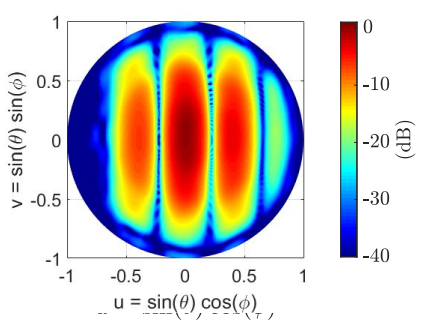

(f)
Fig. 8. Amplitude and phase of the computed equivalent currents distribution obtained using the proposed system (a) and (b), respectively, and employing a standard planar range (c) and (d), respectively. UV representation of the retrieved antenna radiation pattern obtained using the proposed system (e) and employing a standard planar range (f).

The amplitude of the reconstructed equivalent currents distribution at $32 \mathrm{GHz}$ is depicted in Fig. 8a and Fig. 8b using the measurements obtained with the proposed system and with the standard planar range, respectively. As it can be seen, although small artifacts below $-20 \mathrm{~dB}$ appear when using the proposed system, the results are similar. The same holds for the phase of the reconstructed equivalent currents distribution, which is shown in Fig. 8c and Fig. 8d using the proposed system and the planar range, respectively. The radiation pattern computed 


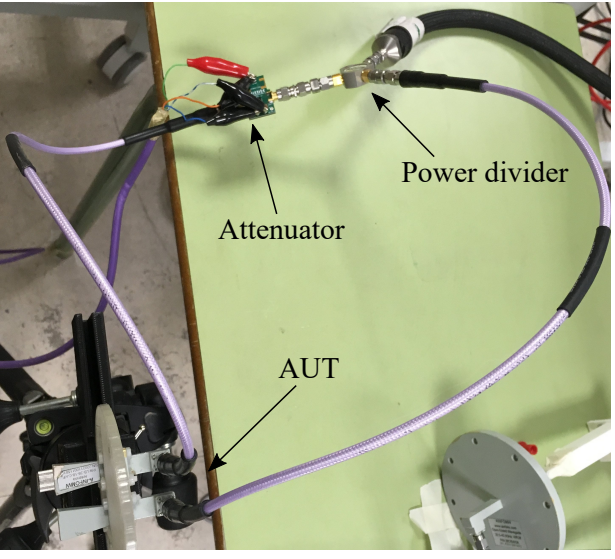

Fig. 9. Measurement setup using an attenuator connected to one of the horns of the two antenna array.

from the equivalent currents distribution estimated using the proposed system and the planar range is depicted in Fig. 8e and Fig. 8f, respectively. As it can be observed, although the pattern obtained with the proposed system is noisier than the one measured with the planar range, the main and the secondary lobes are well-reconstructed, which demonstrates the accuracy of the proposed system.

\section{B. Two horn antenna array with an attenuator}

For the next test a variable attenuator was added to one element of the previous two horn antenna array (Fig. 9). In this case the VNA was configured to acquire 201 frequency points between 24 and $32 \mathrm{GHz}$ and the same probe antenna as in the former experiment was used. During the scan a total of 7271 acquisitions were performed at an average distance of $3.6 \mathrm{~cm}$ from the AUT and the size of the cuboids used to balance the sampling were given by $d=2 \mathrm{~mm}$ and $t=5 \mathrm{~mm}$.

Results for $f=32 \mathrm{GHz}$ are shown in Fig. 10. At this frequency, the attenuator level, previously measured using the VNA, is $4.6 \mathrm{~dB}$. This level matches the difference existing between the amplitude values of the reconstructed equivalent currents on each horn antenna aperture (Fig. 10a), which results in $4.5 \mathrm{~dB}$. In addition, the phase shift between the reconstructed currents on each aperture is $55^{\circ}$ (Fig. 10b), in agreement with the extra phase shift of the attenuator branch measured with the VNA. The heatmap depicting where measurements were taken is shown in Fig. 10c and the Hplane cut of the far-field pattern computed through a NF-FF transformation is shown in blue in Fig. 10d, where a good correspondence in the main and side lobes with a theoretical model can be observed; higher differences appear for values of other secondary lobes of the pattern below $-20 \mathrm{~dB}$.

The difference in both magnitude and phase of the maximum of the equivalent currents distribution between both horn antennas is depicted for all the measured frequencies in Figs. $11 \mathrm{a}$ and $11 \mathrm{~b}$, respectively. In addition, the same difference, depicted in red, was characterized using the VNA measuring directly the $S_{21}$ parameter between the end ports of the array feeding network. As it can be seen in Fig. 11a, the difference in magnitude obtained with the diagnosis system and from

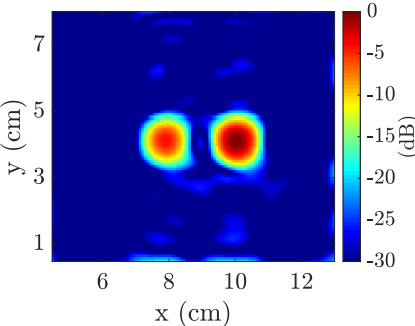

(a)

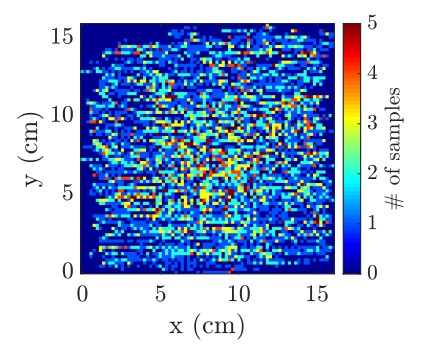

(c)

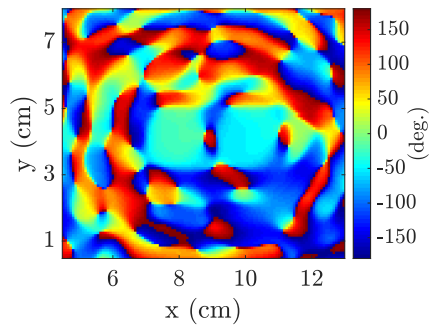

(b)

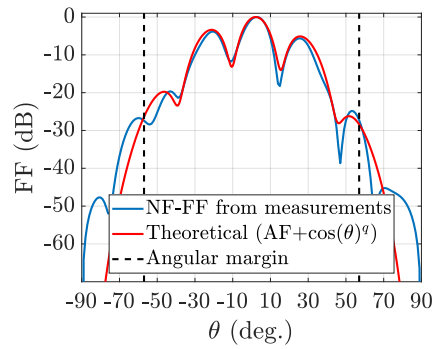

(d)
Fig. 10. Magnitude (a) and phase (b) of the equivalent currents distribution retrieved from the NF measurements that were acquired at the positions depicted in the heatmap (c). The H-plane cut of the far-field pattern computed through a NF-FF transformation is shown in (d).

the feeding network measurement are very similar with errors below $1 \mathrm{~dB}$. The curve obtained with the proposed antenna measurement system presents a higher ripple, probably due to the presence of reflections in the near environment. The phase difference retrieved using the system is very similar to that obtained using the VNA across the whole measured frequency range as can be observed in Fig. 11b. As it can be seen, in $97 \%$ of the cases the error is below $5^{\circ}$, being the maximum error $7.8^{\circ}$.

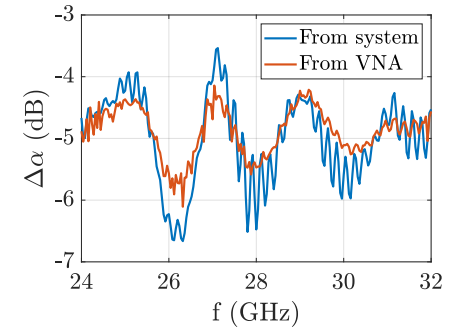

(a)

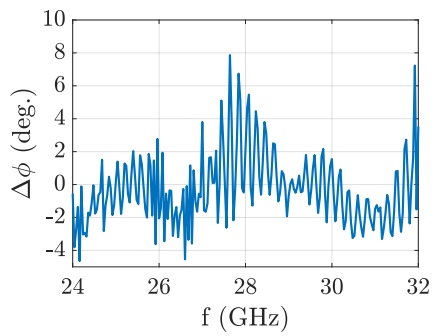

(b)
Fig. 11. Difference in magnitude between the maximum of the equivalent currents distribution corresponding to each horn of the array (a). The value retrieved using the proposed system is depicted in blue and the difference obtained using the VNA is depicted in red. The error between the phase values of that same difference obtained using the VNA and the proposed system is depicted in b).

\section{Leaky-wave antenna}

Finally, the leaky-wave antenna depicted in Fig. III-C was diagnosed at $12 \mathrm{GHz}$ with the proposed system, using both the PNA-X and the Fieldfox portable VNA. In addition, this antenna was also measured in a conventional planar range [35] in order to have a reference result. The probe antenna was an open-ended waveguide WR90. 
Using the proposed system and the PNA-X a total of 937 $\mathrm{NF}$ samples were acquired. Measurements were taken at an average distance of $11 \mathrm{~cm}$ from the AUT, covering an area of $27 \times 33 \mathrm{~cm}$, and the size of the cuboids used to balance the sampling were given by $d=1 \mathrm{~cm}$ and $t=1 \mathrm{~cm}$.

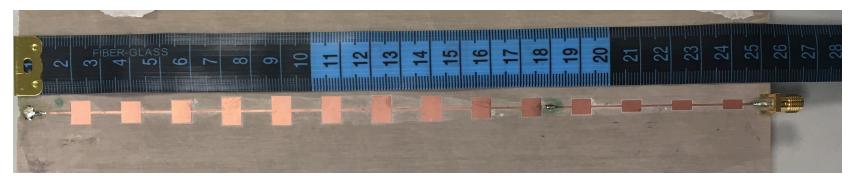

Fig. 12. Measured leaky-wave antenna.

Concerning the measurements using the planar range with the setup depicted in Fig. 13, a total of 10201 NF samples were acquired in a planar domain of $1 \times 1 \mathrm{~m}$, with a sampling step of $1 \mathrm{~cm}$ in the $\mathrm{x}$ - and $\mathrm{y}$ - axes.

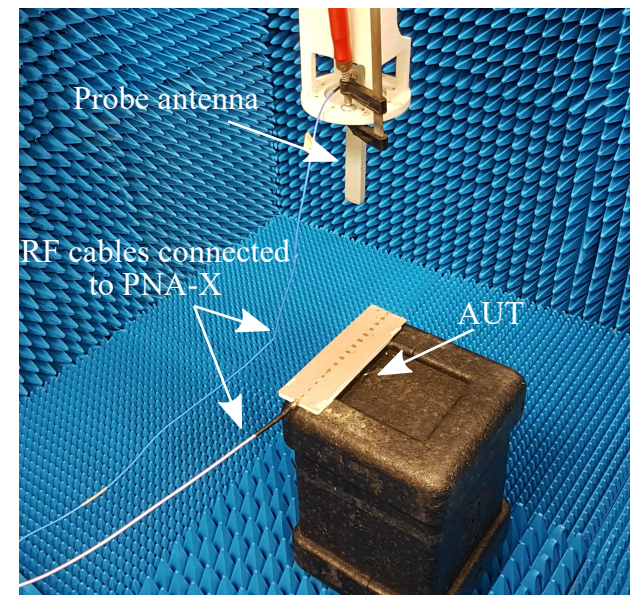

Fig. 13. Measurement setup in the planar range.

The magnitude of the equivalent currents distribution obtained using the proposed system and the planar range are depicted in Figs. 14a and 14b, respectively. As it can be seen, the shape and magnitude of the currents are in good agreement. In addition, in order to show the influence of the value of $d$, the NF samples acquired with the proposed system were postprocessed using $d=2 \mathrm{~cm}(0.8 \lambda)$. The computed equivalent currents distribution are also depicted in Fig. 14c, where it can be noticed how the reconstructed equivalent currents distribution exhibits more artifacts.

The phase of the equivalent currents distribution retrieved with the proposed system and with the planar range are depicted in Figs. 15a and 15b, respectively. For a better comparison, only the phase where the magnitude of the equivalent currents distribution is above $-15 \mathrm{~dB}$ is plotted. As it can be observed, both phase profiles are in good agreement.

Finally, the far-field pattern was computed from the equivalent currents distribution by means of a NF-FF transformation. In particular, the results of the E-plane cut obtained using the proposed system with the PNA-X, the proposed system with the Fieldfox portable VNA as well as using the planar range are depicted in Fig. 16. Using the Fieldfox portable VNA a total of 764 samples were acquired at an average distance of $10.3 \mathrm{~cm}$, covering an area of $26 \times 31 \mathrm{~cm}$. In this example, it can be noticed that the proposed system, using both hardware configurations, is able to correctly compute the position of the maximum of the field pattern at $\theta=-22^{\circ}$. In addition, the $-15 \mathrm{~dB}$ sidelobe at $\theta=20^{\circ}$ is also predicted and the rest of radiation pattern in the angular margin of validity (denoted with vertical dashed lines in Fig. 16) is in a fair agreement with the planar range results despite the freehand measurements not being accomplished in an anechoic environment.

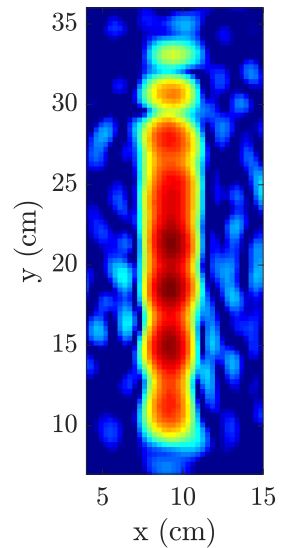

(a)

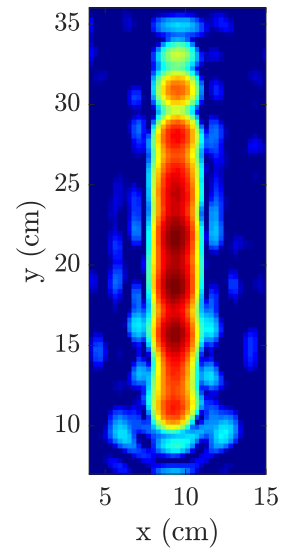

(b)

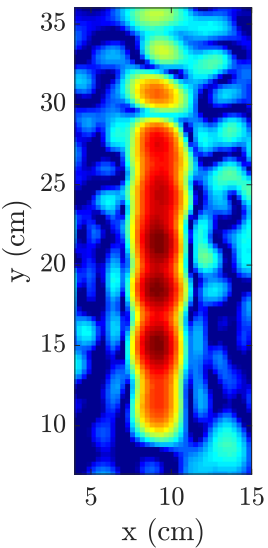

(c)

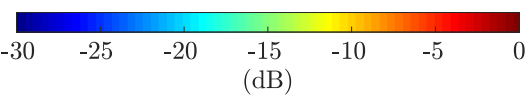

Fig. 14. Magnitude of the computed equivalent currents distribution obtained using the proposed system and $d=1 \mathrm{~cm}$ (a), using a planar range (b) and employing the proposed system and $d=2 \mathrm{~cm}$ (c).

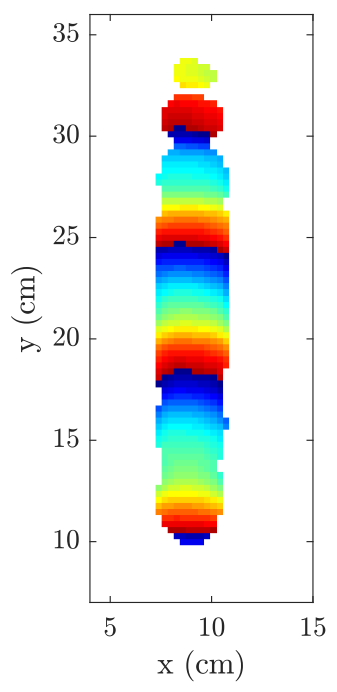

(a)

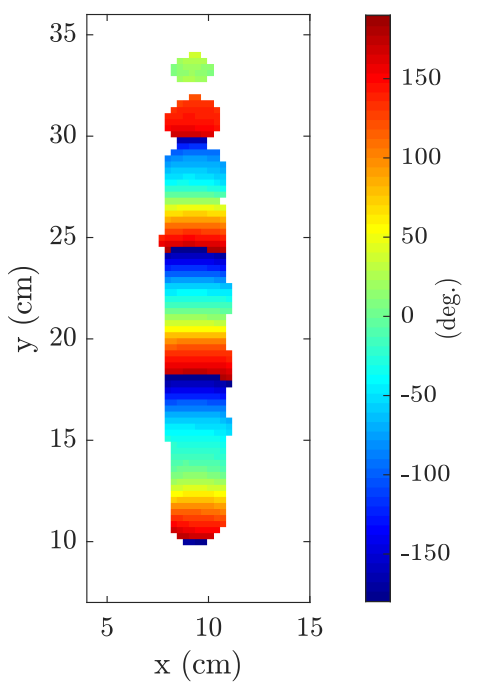

(b)
Fig. 15. Phase of the equivalent currents distribution retrieved from the NF measurements obtained using the proposed system (a) and using a planar range (b). Only the phase where the magnitude of the equivalent currents distribution is above $-15 \mathrm{~dB}$ is plotted.

\section{CONCLUSIONS}

A system to perform freehand scanning of antennas providing fast diagnosis without the need of a typical antenna measurement range has been presented. The system comprises 


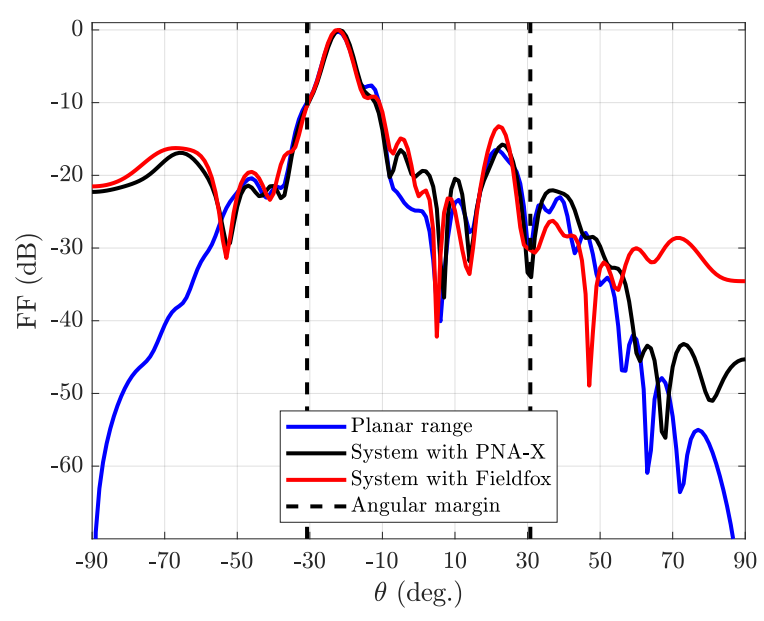

Fig. 16. Measured E-plane cut of the radiation-pattern of the leaky-wave antenna.

a handheld probe antenna, which is moved in front of the AUT aperture, a VNA, which is connected to both the probe antenna and the AUT, a motion capture system to retrieve the position of the probe antenna, and a conventional laptop in charge of controlling the field and position samples and of processing the acquired data. During the scan, field and position acquisitions are triggered as the operator moves the antenna in front of the AUT and the SRM is applied to the obtained data to provide a real-time estimation of an equivalent currents distribution on the aperture of the AUT and its radiation pattern after a NF-FF transformation is performed. In addition, several strategies to cope with non regular hand movements of the probe antenna, which result in an uneven distribution of field samples, are discussed.

The proposed system was validated through several sets of measurements in $\mathrm{Ku}$ and $\mathrm{Ka}$ bands. The results show that a fast and rough diagnosis of the status of radiating elements can be obtained in a few seconds while a full and more accurate characterization of antennas can be achieved in only a few minutes for low to medium size antennas. Although the accuracy is not expected to be as high as the one obtained in typical anechoic antenna measurement ranges, the results obtained with the presented system exhibit very good accuracy at mm-wave frequencies.

In addition, the presented system can be deployed in a matter of minutes and it can be easily implemented with portable components, paving the way to a new approach to perform fast, in situ diagnosis. Future work will focus on modifying the system to include phaseless algorithms to avoid the need of a connection to the antenna under test as well as to reduce the overall cost.

\section{REFERENCES}

[1] O. M. Bucci, A. Capozzoli, and G. D'Elia, "Diagnosis of array faults from far-field amplitude-only data," IEEE Transactions on Antennas and Propagation, vol. 48, no. 5, pp. 647-652, May 2000.

[2] J. A. Rodriguez-Gonzalez, F. Ares-Pena, M. Fernandez-Delgado, R. Iglesias, and S. Barro, "Rapid method for finding faulty elements in antenna arrays using far field pattern samples," IEEE Transactions on Antennas and Propagation, vol. 57, no. 6, pp. 1679-1683, June 2009.
[3] G. Oliveri, P. Rocca, and A. Massa, "Reliable diagnosis of large linear arrays-a bayesian compressive sensing approach," IEEE Transactions on Antennas and Propagation, vol. 60, no. 10, pp. 4627-4636, Oct 2012.

[4] M. D. Migliore, "A compressed sensing approach for array diagnosis from a small set of near-field measurements," IEEE Transactions on Antennas and Propagation, vol. 59, no. 6, pp. 2127-2133, June 2011.

[5] Y. Alvarez, F. Las-Heras, and M. R. Pino, "Reconstruction of equivalent currents distribution over arbitrary three-dimensional surfaces based on integral equation algorithms," IEEE Transactions on Antennas and Propagation, vol. 55, no. 12, pp. 3460-3468, Dec 2007.

[6] J. Araque and G. Vecchi, "Field And Source Equivalence In Source Reconstruction On 3D Surfaces," Progress In Electromagnetics Research, vol. 103, pp. 67-100, 2010.

[7] K. Persson and M. Gustafsson, "Reconstruction of equivalent currents using a near-field data transformation with radome applications," Progress In Electromagnetics Research, vol. 54, pp. 179-198, 2005.

[8] F. Las-Heras and T. K. Sarkar, "A direct optimization approach for source reconstruction and $\mathrm{nf}$-ff transformation using amplitude-only data," IEEE Transactions on Antennas and Propagation, vol. 50, no. 4, pp. 500-510, April 2002.

[9] F. Cano, M. Sierra-Castañer, S. Burgos, and J. L. Besada, "Applications of sources reconstruction techniques: Theory and practical results," in Proceedings of the Fourth European Conference on Antennas and Propagation, April 2010, pp. 1-5.

[10] J. Araque and G. Vecchi, "Removal of unwanted structural interactions from antenna measurements," in 2009 IEEE Antennas and Propagation Society International Symposium, June 2009, pp. 1-4.

[11] L. J. Foged, L. Scialacqua, F. Saccardi, J. L. A. Quijano, G. Vecchi, and M. Sabbadini, "Practical application of the equivalent source method as an antenna diagnostics tool [amta corner]," IEEE Antennas and Propagation Magazine, vol. 54, no. 5, pp. 243-249, Oct 2012.

[12] Y. Alvarez, F. Las-Heras, M. R. Pino, and J. A. Lopez, "Acceleration of the sources reconstruction method via the fast multipole method," in 2008 IEEE Antennas and Propagation Society International Symposium, July 2008, pp. 1-4.

[13] T. F. Eibert and C. H. Schmidt, "Multilevel fast multipole accelerated inverse equivalent current method employing rao-wilton-glisson discretization of electric and magnetic surface currents," IEEE Transactions on Antennas and Propagation, vol. 57, no. 4, pp. 1178-1185, April 2009.

[14] Y. Alvarez, F. Las-Heras, and M. R. Pino, "Application of the adaptive cross approximation algorithm to the sources reconstruction method," in 2009 3rd European Conference on Antennas and Propagation, March 2009, pp. 761-765.

[15] J. A. Lopez-Fernandez, M. Lopez-Portugues, Y. Alvarez-Lopez, C. Garcia-Gonzalez, D. Martinez, and F. Las-Heras, "Fast Antenna Characterization Using the Sources Reconstruction Method on Graphics Processors," Progress In Electromagnetics Research, vol. 126, pp. 185201, 2012

[16] H. He, "The development of near field probing systems for EMC near field visualization and EMI source localization," Master's thesis, Missouri University of Science and Technology, 2015. [Online]. Available: https://scholarsmine.mst.edu/masters_theses/7435

[17] H. He, P. Maheshwari, and D. J. Pommerenke, "The Development of an EM-Field Probing System for Manual Near-Field Scanning," IEEE Transactions on Electromagnetic Compatibility, vol. 58, no. 2, pp. 356363, April 2016.

[18] H. He, V. Khilkevich, and D. Pommerenke, "2D imaging system with optical tracking for EMI source localization," in 2015 IEEE Symposium on Electromagnetic Compatibility and Signal Integrity, March 2015, pp. $107-110$.

[19] G. Álvarez-Narciandi, M. López-Portugués, F. Las-Heras, and J. Laviada, "Freehand, agile, and high-resolution imaging with compact $\mathrm{mm}$ wave radar," IEEE Access, vol. 7, pp. 95 516-95 526, 2019.

[20] P. Petre and T. K. Sarkar, "Planar near-field to far-field transformation using an equivalent magnetic current approach," IEEE Transactions on Antennas and Propagation, vol. 40, no. 11, pp. 1348-1356, Nov 1992.

[21] Y. Alvarez Lopez, F. Las-Heras Andres, M. R. Pino, and T. K. Sarkar, "An improved super-resolution source reconstruction method," IEEE Transactions on Instrumentation and Measurement, vol. 58, no. 11, pp. 3855-3866, Nov 2009.

[22] C. H. Schmidt and T. F. Eibert, "Assessment of irregular sampling nearfield far-field transformation employing plane-wave field representation," IEEE Antennas and Propagation Magazine, vol. 53, no. 3, pp. 213-219, June 2011.

[23] M. Farouq, M. Serhir, and D. Picard, "Matrix method for far-field calculation using irregular near-field samples for cylindrical and spherical 
scanning surfaces," Progress In Electromagnetics Research B, vol. 63, pp. 35-48, 2015.

[24] _ , Matrix method for antenna plane wave spectrum calculation using irregularly distributed near-field data: Application to far-field assessment," Progress In Electromagnetics Research M, vol. 42, pp. 7183, 2015.

[25] Y. Alvarez, F. Las-Heras, and M. R. Pino, "On the comparison between the spherical wave expansion and the sources reconstruction method," IEEE Transactions on Antennas and Propagation, vol. 56, no. 10, pp. $3337-3341$, Oct 2008.

[26] A. Geise, O. Neitz, J. Migl, H. Steiner, T. Fritzel, C. Hunscher, and T. F. Eibert, "A crane based portable antenna measurement system system description and validation," to appear in IEEE Transactions on Antennas and Propagation, pp. 1-1, 2019.

[27] M. García-Fernández, Y. Álvarez López, A. Arboleya, B. GonzálezValdés, Y. Rodríguez-Vaqueiro, M. E. De Cos Gómez, and F. Las-Heras Andrés, "Antenna diagnostics and characterization using unmanned aerial vehicles," IEEE Access, vol. 5, pp. 23 563-23 575, 2017.

[28] G. Virone, A. M. Lingua, M. Piras, A. Cina, F. Perini, J. Monari, F. Paonessa, O. A. Peverini, G. Addamo, and R. Tascone, "Antenna pattern verification system based on a micro unmanned aerial vehicle (uav)," IEEE Antennas and Wireless Propagation Letters, vol. 13, pp. 169-172, 2014.

[29] G. Álvarez-Narciandi, J. Laviada, Y. Álvarez-López, and F. Las-Heras, "Rapidly Deployable Portable System for Real-Time Antenna Diagnostics and Characterization," in 2019 IEEE International Symposium on Antennas and Propagation and USNC-URSI Radio Science Meeting, July 2019, pp. 1689-1690.

[30] OptiTrack. (2019, May) Optitrack motion capture system. [Online]. Available: http://www.optitrack.com

[31] "IEEE Recommended Practice for Near-Field Antenna Measurements," IEEE Std 1720-2012, pp. 1-102, Dec 2012.

[32] "IEEE Standard Test Procedures for Antennas," ANSI/IEEE Std 1491979, 1979.

[33] C. A. Balanis, Advanced Engineering Electromagnetics. Wiley, 2010.

[34] S. Gregson, Principles of Planar Near-Field Antenna Measurements, ser. Electromagnetic Waves. Institution of Engineering and Technology, 2007. [Online]. Available: https://digitallibrary.theiet.org/content/books/ew/pbew053e

[35] A. Arboleya, Y. Álvarez, and F. Las-Heras, "Millimeter and submillimeter planar measurement setup," in 2013 IEEE Antennas and Propagation Society International Symposium (APSURSI), July 2013, pp. 1-2.

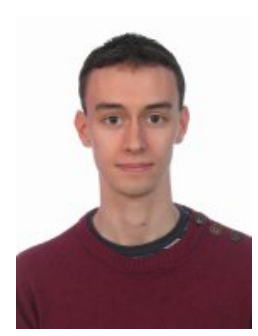

Guillermo Alvarez-Narciandi Guillermo ÁlvarezNarciandi received the M.Sc. degree in Telecommunication Engineering from the University of Oviedo, Gijón, Spain, in 2016, where he is currently working towards the Ph.D. degree (with the support of a FPU grant from the Spanish Government). He was a Visiting Student at Stanford University, CA, USA, in 2014, and a visiting scholar at the University of Pisa (Italy) in 2018 and at the Institute of Electronics, Microelectronics and Nanotechnology (IEMN), University of Lille (France) in 2019. He received the AMTA 2019 Student Paper Award (second place) and the Special Award to the Best Entrepreneurship Initiative in the XV Arquímedes national contest in 2017 for the development of a RFID-based location system. His main research interests are radar systems and imaging techniques, antenna diagnosis and characterization systems, localization and attitude estimation systems and RFID technology.

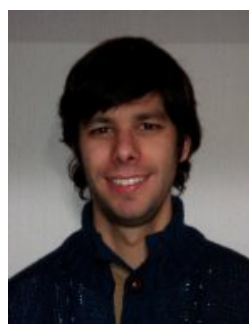

Jaime Laviada Jaime Laviada was born in Gijón, Spain. He received the M.S. degree in telecommunication engineering and the Ph.D. degree from the Universidad de Oviedo, Spain (http://www.uniovi.es), in 2005 and 2010, respectively. In 2006, he joined the research group Signal Theory and Communications of the Universidad de Oviedo, where he has been involved in multiple national and European projects as well as contracts with several companies. In 2015, he moved to the Antennas Group of the Universidad Pública de Navarra with a national postdoctoral fellowship collaborating in several applied research projects. Finally, he moved back to the Universidad de Oviedo where he currently holds a position of associate professor. In addition, he has been a Visiting Scholar in the Electromagnetics and Communications Lab, Pennsylvania State University, during 2007 and 2008 as well as in the Applied Microwave NonDestructive Testing Laboratory, Missouri S\&T, during 2017. His research interests include numerical techniques applied to EM imaging, antenna measurements, method of moments, and antenna pattern synthesis.

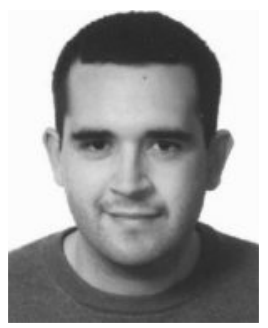

Yuri Alvarez-López Yuri Álvarez López (S'06-M'09-SM'15) was born in Langreo, Spain, in 1983. He received the M.S. and Ph.D. degrees in telecommunication engineering from the Universidad de Oviedo, Gijón, Spain, in 2006 and 2009, respectively. He was a Visiting Scholar with the Department of Electrical Engineering and Computer Science, Syracuse University, Syracuse, NY, USA, in 2006 and 2008, respectively, held a visiting post-doctoral position at the Gordon Center for Subsurface Sensing and Imaging Systems Awareness and Localization of Explosive Related Threats Center of Excellence, Northeastern University, Boston, MA, USA, from 2011 to 2014 and held a visiting post-doctoral position at the ELEDIA Research Center, Trento, Italy, in 2015. He is currently a Professor with the Signal Theory and Communications, Universidad de Oviedo. His research interests include antenna diagnostics, antenna measurement techniques, RF techniques for indoor location, inverse scattering and imaging techniques, and phaseless methods for antenna diagnostics and imaging. He was a recipient of the 2011 Regional and National Awards to the Best Ph.D. Thesis on Telecommunication Engineering (category: security and defense).

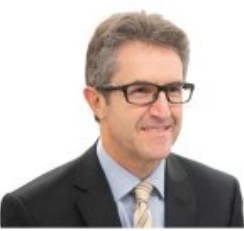

Fernando Las-Heras Fernando Las-Heras received the M.S. in 1987 and the Ph.D. in 1990, both in Telecommunication Engineering, from the Technical University of Madrid (UPM). He was a National Graduate Research Fellow (1988-1990) and he held a position of Associate Professor at the Dept. of Signal, Systems and Radiocommunications of the UPM (1991-2000). From 2003 he holds a Full-Professor position at the University of Oviedo where he was the Vice-dean for Telecommunication Engineering at the Technical School of Engineering at Gijón (2004-2008). As of 2001 he heads the research group Signal Theory and Communications TSC-UNIOVI at the Dept. of Electrical Engineering of the University of Oviedo. He was a Visiting Lecturer at the National University of Engineering in Peru in 1996, a Visiting Researcher at Syracuse University, New York, in 2000, and a short-term Visiting Lecturer at ESIGELEC in France from 2005 to 2011. He held the Telefónica Chair on "RF Technologies", "ICTs applied to Environment" and "ICTs and Smart cities" at the University of Oviedo (2005-2015). Member of the board of directors of the IEEE Spain Section (2012-2015), member of the board IEEE Microwaves \& Antennas Propagation Chapter (AP03/MTT17) (2016-2017), member of the Science, Technology and Innovation Council of Asturias (2010-12), and president of the professional association of Telecommunication Engineers at Asturias. $\mathrm{He}$ has authored scientific papers in the areas of antennas, EM scattering, metamaterials and inverse problems with application to antenna measurement (NF-FF, diagnostics and holography), electromagnetic imaging (security and NDT) and localization, developing computational electromagnetics algorithms and technology on microwaves, millimeter wave and $\mathrm{THz}$ frequency bands. 